\title{
Generic Wrapper Based Model using Haralick Features for Silk Fabric Defect Classification
}

\author{
Ms. Shweta Loonkar ${ }^{1}$, Dhirendra S. Mishra ${ }^{2}$, and Surya S. Durbha ${ }^{3}$ \\ ${ }^{1}$ Ph.D., Research Scholar, NMIMS University \\ Computer Engineering Department, MPSTME, Mumbai-56 \\ ${ }^{2}$ Professor, NMIMS University \\ Computer Engineering Department, MPSTME, Mumbai-56 \\ ${ }^{3}$ Professor, IIT, Mumbai \\ CSRE, IIT Bombay Powai-76 \\ shwetaloonkar@gmail.com, dhirendra.mishra@gmail.com, sdurbha@iitb.ac.in
}

\begin{abstract}
Quality control unit of fabric industry looks for the effective defect detection methodology. The research is required to be done in this area to develop such solution. Various models based on combination of suitable feature extraction, selection and classification approaches need to be experimented out for the same. This paper attempts to experiment and provide such models mainly based on generic wrapper based selection approaches. Widely used broader range of Haralick features are prominently used for detection and classification of defects in this research. It also attempts to identify the suitability of these features based on segmented images provided as an input. The research has been carried on TILDA Dataset consisting of 800 Silk Fabric Images with eight different defects present on it and each carrying 100 images per defect. Models generated using generic wrapper based approach has also been compared with the Gabor Transforms. Then identification of suitable Haralick Features for particular type of defects has been carried out. In this 68\% classification accuracy has been achieved using generic wrapper method and $40 \%$ accuracy has been achieved using Gabor Transform with respect to fourteen Haralick Features and seven types of defects.
\end{abstract}

\section{Keywords: Generic Wrapper Method, Gabor Transform, Haralick Features, TILDA Dataset.}

\section{INTRODUCTION}

Producing defect free material from factories, warehouses, etc is a complex task. There are many processes involved to get the finished textile products and each stage. There have been enlarging the number of losses due to the defects at each level of processing of fabric [1]. The productivity and the nature of the textile delivered by each industry depend upon the type of defects measured amid the quality control [2]. Numerous endeavours have been made to unravel these problems. As soon as fabric images are obtained they undergo a series of image processing methods like image enhancement, restoration, segmentation, feature extraction, and defect detection [3]. Based on the literature survey done various methods have been deployed for fabric defect detection and classification over the decades.

Statistical, spectral, and model-based approaches have all been presented to solve the challenge of detecting flaws in woven fabrics, with spectral approaches being the most successful detection approaches for woven fabrics. A Gabor transform or filter is one of the most prominent spectral techniques and has been effectively and widely used in the field of defect detection because it has optimal localization both in the spatial domain and in the spatial-frequency domain [4]. All Gabor function-based detection algorithms can be divided into two groups. One is to use a filter bank with unsupervised learning [4-5] and other is an optimal filter with supervised learning [6-7]. Filtering with a filter bank can generate a lot of data for processing, but a series of filters can help in the segmentation of data. 
The selection of relevant attributes from the original images to minimize the redundant features is called the feature selection method [8-9]. The more appropriate the selection of features the performance of the classification is improved. Furthermore, a new paradigm for segmentation and classification uses the wrapper-based approach for feature selection and feature extraction. Various types of flaws like holes, cuts, oil stains, etc may get introduced in the fabric intentionally or unintentionally. So it is need of the hour to understand the relationship between the fabric material and defect types. It performs the look within the space of highlight subsets such as classification performances on cross-validation of the training set which gives better outcome than filter methods using evaluation metric. But wrapper methods increase the computational cost [10-15]. In addition, the best feature subset selection method can reduce the cost of feature measurement.

The important contributions of our research to the literature are as follows:

1. To reduce the number of features using generic wrapper-based methods to generate the predictive models for each defective class using Haralick Features and measure their performance based on Classification Accuracy (CA), Precision, Recall and Kappa Coefficients (KC)

2. Compare the results obtained from the wrapper strategy with approach like Gabor Transforms..

The remaining sections of this writing are structured as follows: Section 2 outlines the basic working principles of the wrapper-based approach, and then Section 3 describes the proposed methodology used. Next, section 4 presents an experimental framework for the result and discussion obtained from the proposed algorithm and compares it with the other classification and segmentation methods. Finally, section 5 concludes the paper with possible future work.

\section{OVERVIEW OF WRAPPER BASED APPROACH}

The Feature Selection (FS) approach is a commonly used method for diminishing dimensionality of the information and expanding the proficiency of learning calculation. For FS we look up the whole search space. The possible subsets of features are selected using an equation 1 [15]:

$$
\sum_{s=0}^{n}\left(\begin{array}{l}
n \\
s
\end{array}\right)=\left(\begin{array}{l}
n \\
0
\end{array}\right)+\left(\begin{array}{l}
n \\
1
\end{array}\right)+\left(\begin{array}{l}
n \\
2
\end{array}\right) \ldots\left(\begin{array}{l}
n \\
n
\end{array}\right)=2^{n}
$$

Where:

$\mathrm{n}=$ Number of features present

$\mathrm{s}=$ Current feature subset size

FS methods can be grouped into four major categories:

- Filter Approach: If FS is done without using a learning algorithm, then it is known as the filter approach. It uses the inter-class separable principle for selecting and evaluating features [16].

- Wrapper Approach: If the FS approach is done using a classification algorithm, then it is called the wrapper approach. In this method, the selected features are fed into the learning model to measure the performance of the selected features [17]. The wrapper approach is constituted by three components as shown in Figure 1.

$\circ \quad$ Learning Machine

- Criteria used for Feature Evaluation

$\circ \quad$ Feature Selection Method 


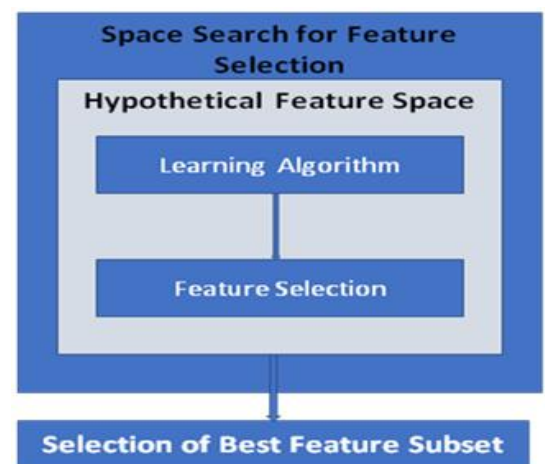

Figure 1. Basic Principle used for Wrapper Approach

1. Embedded Approach: This method is almost similar to the wrapper-based approach, but here the link between the FS and classifier is stronger [18].

2. Hybrid Approach: Hybrid methods are the combination of any methods mentioned above. Some work has suggested two-step feature selection methods [19]. In this number of features are selected using the first method and the second method is used to reduce them. This method is used to attain better results.

\section{PROPOSED METHODOLOGY}

This section explains the methodology used by the wrapper-based approach for feature selection from silk images present in TILDA Database. This method was validated by processing it for multiple images of the different defects like Punches (D1), Oil Stains (D2), Thread Condensation (D3), Wrinkles (D4), Camera Distortion (D5), Foreign Body (D6), and Lightning Condition (D7). As a result, the suggested system will determine which classification approach; Haralick Feature, and Defect Type yields the best results while running the model, and vice versa.

\subsection{Tilda Database:}

The data set contains 800 images of silk material. All images are monochrome gray images with 256 grayscale. For each class, there are 100 images as shown in Figure 2. The images are saved in TIFF format and have a dimension of $768 * 512$ pixels [20].

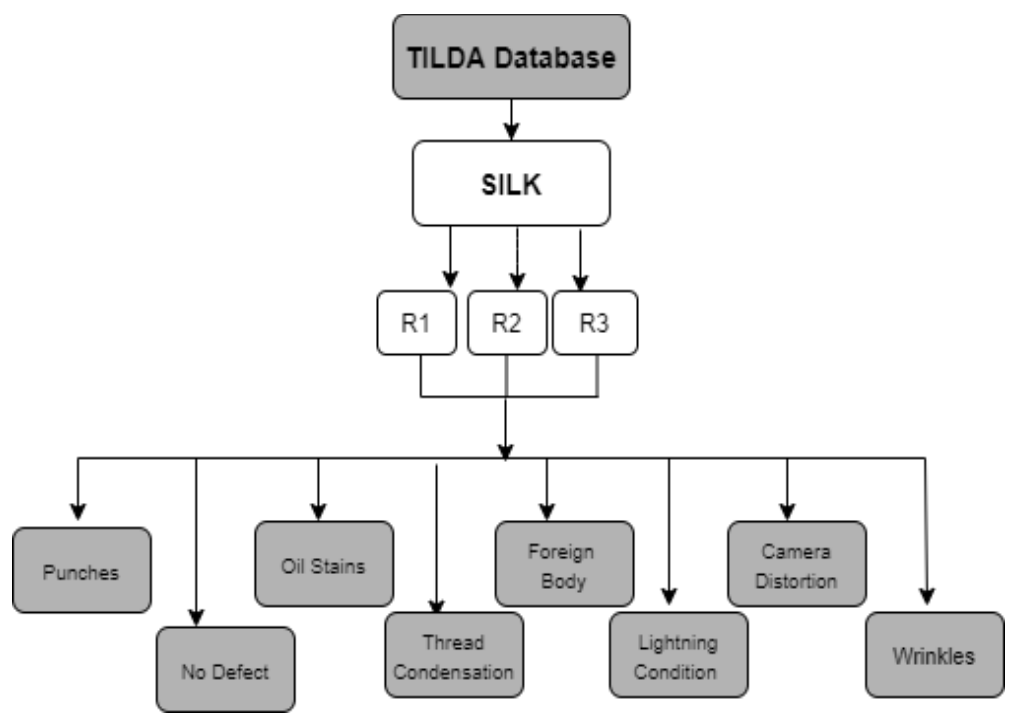

Figure 2. TILDA Database for Experimentation [20] 


\subsection{Generic Wrapper Based Method:}

The general model used for the wrapper-based method is shown in figure 3. Below mentioned are the steps which show how wrapper methods work [19]:

- Look for a specific set of characteristics: We select a subset of features from the available ones using a search approach.

- Construct a machine-learning model: In this step, a pre-selected subset of features is used to train a machine-learning algorithm.

- Examine the model's performance: Finally, we use a chosen metric to evaluate the newlytrained ML model.

- Repeat: The entire process is restarted with a new set of features, a new machine learning model, and so on.

This process goes on until the desired condition is met and then, we choose the best subset with the best outcome.

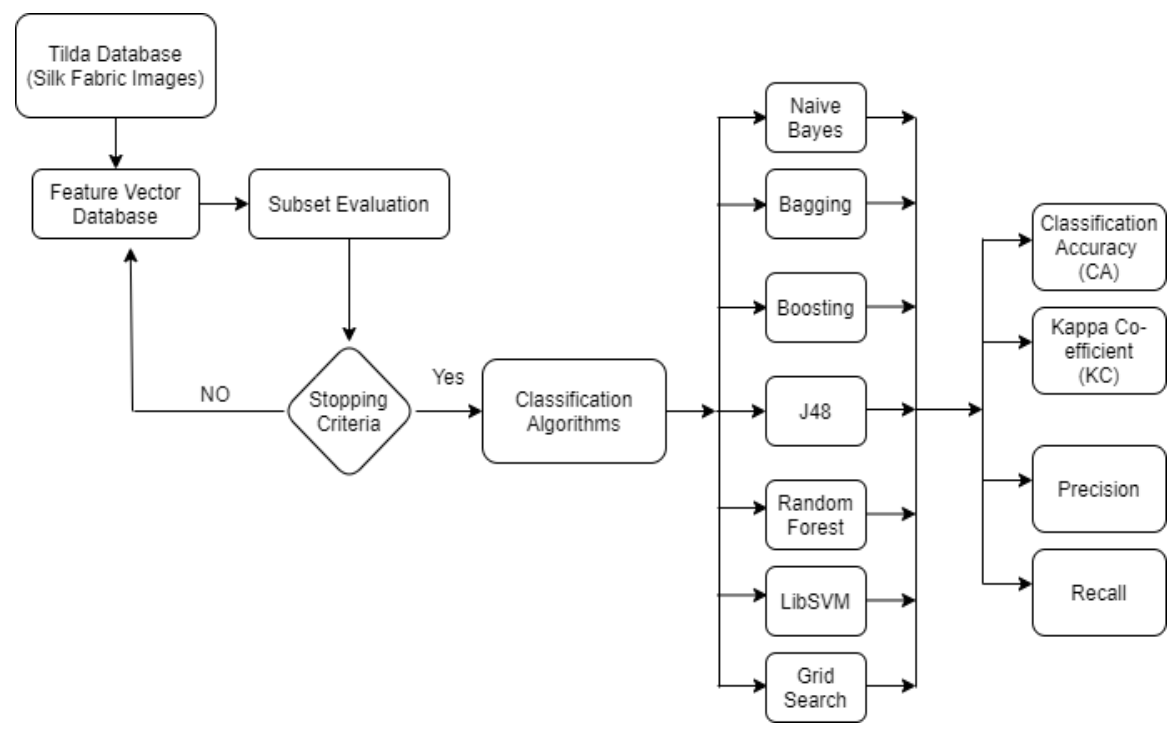

Figure 3. A Unified Model for Feature Selection and Extraction

The "wrapper" approach encases a classifier in a full training set and cross-validation loop, searching the attribute space for an appropriate feature set using the classifier. Starting from any subset, we can search forwards, backward, or bidirectional. The segmentation methods were used on silk fabric to create the feature vector database. Gray Level Co-Occurrence Matrix (GLCM) with Haralick Feature was used on the segmented image to create FVDB. Below mentioned are the steps for making these models:

- Obtain the segmented image by applying Mean Shift Segmentation.

- Generate Feature Vector Database by applying GLCM using Haralick Features on the segmented image.

- Input the FVDB into the Machine Learning Tool and apply generic wrapper-based approaches: Features are extracted using Wrapper Subset Evaluation and Classifier Subset Evaluation and features are selected and searched using Best-first Search, Greedy Step Wise and Genetic search.

- The generated feature subsets are evaluated using well-known classifiers such as Random Forest (RF), Decision Tree (J48), Bagging, Boosting, and Support Vector Machine (SVM) for validating the output of Feature Selection Algorithms.

- A reduced feature subset is used to train the classifiers and measure their accuracy using various performance metrics like classification accuracy, kappa coefficients precision, and recall. 
Figure 4 shows the block diagram of generic wrapper based approach. The abbreviations used in Figure 4 are stated below in Table 1.

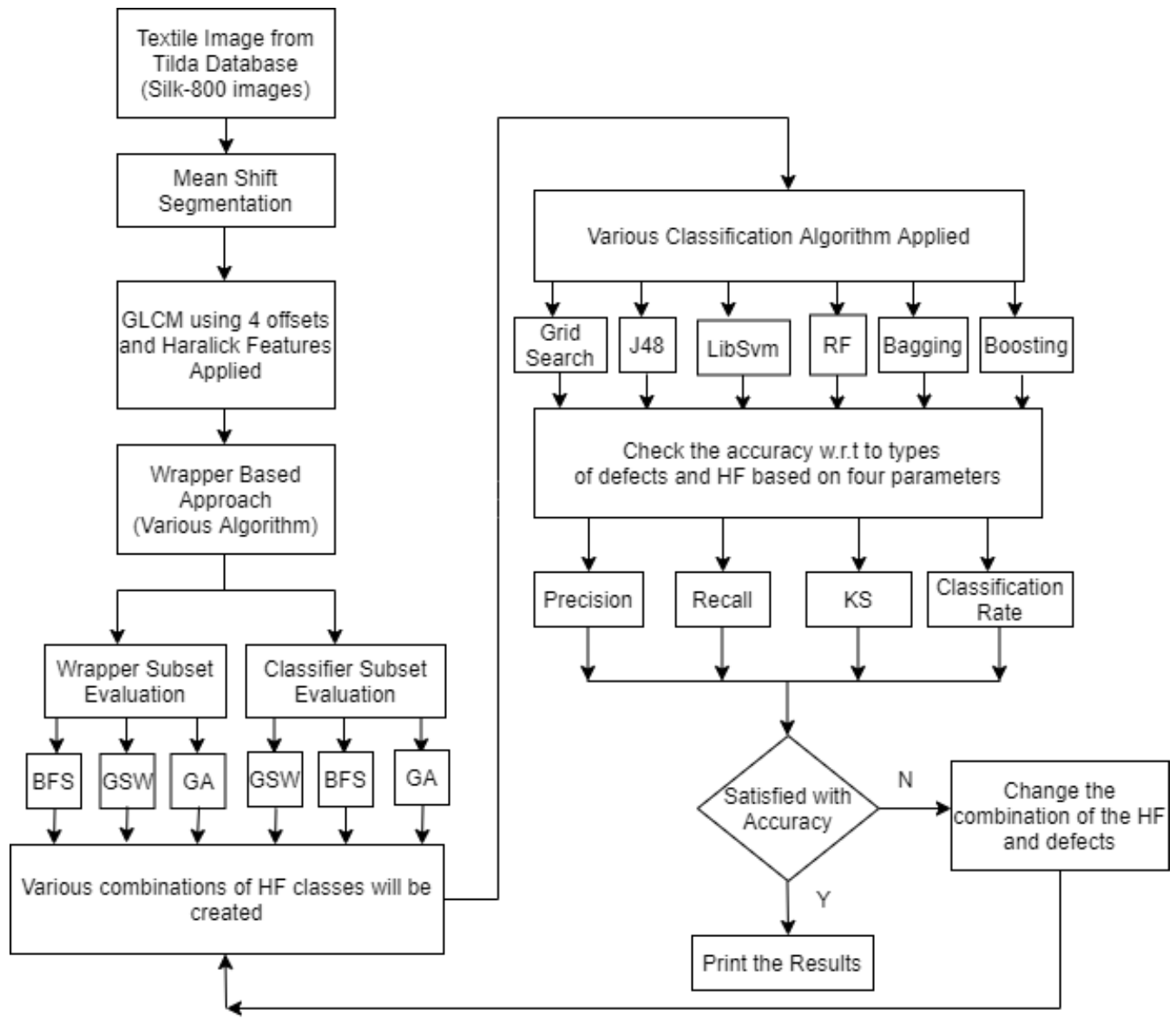

Figure 4. Wrapper Based Approach for Subset Evaluation and Optimal Feature Selection

[ BFS: Breadth-First Search, GSW- Greedy step wise, GA-Genetic Algorithm, RF-Random Forest, HF-Haralick features]

Also the 14 different Haralick Features used in this paper are as: HF1 (Angular Second Moment), HF2 (Contrast), HF3 (Correlation), HF4 (Variance), HF5 (Inverse Difference), HF6 (Sum Average), HF7 (Sum Variance), HF8 (Sum Entropy), HF9 (Entropy), HF10 (Difference Variance), HF11 (Difference Entropy), HF12 (Information Measure of Correlation-I), HF13 (Information Measure of Correlation-II), and HF14 (Maximal Correlation Coefficient).

\subsection{Gabor Transform:}

Gabor Filter is a texture analysis linear filter that determines whether there is any certain frequency content in the image in specific directions in a confined region surrounding the point or region of analysis. Gabor filters and Gabor wavelets are related because they can be built for a variety of dilations and rotations. However expansion is not possible for Gabor Wavelets. As a result, a filter bank made up of Gabor filters of various sizes and rotations is frequently developed.

The algorithm used to extract the features using Gabor Wavelet Transform has been mentioned below and block diagram for the same is shown in figure 5 . 


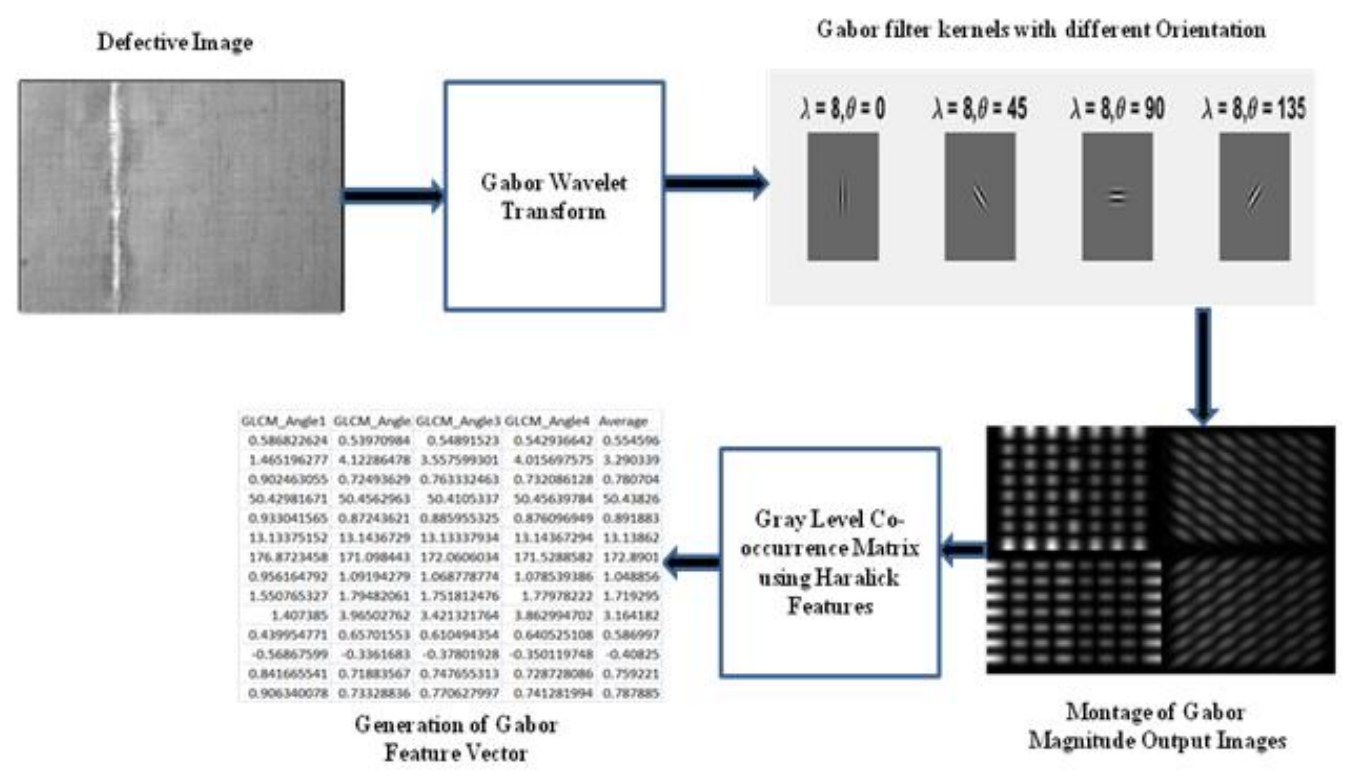

Figure 5 Output Generations at each Step in Gabor Wavelet Transform

\section{RESULTS AND DISCUSSIONS}

In this part, we provide the results of several models generated throughout the learning and testing phase for the development, optimization, and validation of Haralick characteristics appropriate for specific types of errors. This section compares alternative Generic Wrapper and Gabor transform approaches for feature extraction and feature selection methods based on four parameters: classification accuracy, kappa coefficients, precision, and recall. We have conducted an extensive study utilizing a variety of wrapper search algorithms, including best-first search and greedy step-wise. Table 2 and 3 shows the nomenclature used for the Wrapper-based approach and Gabor Transforms.

Table 1. Model Number used for Simple Wrapper Based Approaches

\begin{tabular}{|c|c|c|c|c|c|l|}
\hline S.NO & $\begin{array}{c}\text { Model } \\
\text { Number }\end{array}$ & \multicolumn{1}{|c|}{ Segmentation } & \multicolumn{1}{|c|}{$\begin{array}{c}\text { Feature } \\
\text { Extraction }\end{array}$} & $\begin{array}{c}\text { Feature } \\
\text { Selection }\end{array}$ & $\begin{array}{c}\text { Training } \\
\text { Method }\end{array}$ & $\begin{array}{c}\text { Classification } \\
\text { Algorithm }\end{array}$ \\
\hline 1 & WWG01 & Mean Shift Segmentation & Wrapper Subset Evaluation & Best First Search & Full Training Set & Naïve Bayes \\
\hline 2 & WWG09 & Mean Shift Segmentation & Wrapper Subset Evaluation & Greedy Step Wise & Full Training Set & Bagging \\
\hline 3 & WWG17 & Mean Shift Segmentation & Wrapper Subset Evaluation & Best First Search & 10 Fold CV & Boosting \\
\hline 4 & WWG25 & Mean Shift Segmentation & Wrapper Subset Evaluation & Greedy Step Wise & 10 Fold CV & LibSVM \\
\hline 5 & WWG33 & Mean Shift Segmentation & Classifier Subset Evaluation & Best First Search & Full Training Set & J48 \\
\hline 6 & WWG41 & Mean Shift Segmentation & Classifier Subset Evaluation & Greedy Step Wise & Full Training Set & Random Forest \\
\hline 7 & WWG49 & Mean Shift Segmentation & Classifier Subset Evaluation & Best First Search & 10 Fold CV & Grid Search \\
\hline 8 & WWG56 & Mean Shift Segmentation & Classifier Subset Evaluation & Greedy Step Wise & 10 Fold CV & Grid Search \\
\hline
\end{tabular}

Table 2. Model Number used for Gabor Transform Approaches

\begin{tabular}{|c|l|c|c|l|}
\hline GW01 & Classifier Subset Evaluation & BFS & Full Training Set & Naïve Bayes \\
\hline GW02 & Classifier Subset Evaluation & BFS & Full Training Set & Bagging \\
\hline GW03 & Classifier Subset Evaluation & BFS & Full Training Set & Boosting \\
\hline GW08 & Classifier Subset Evaluation & BFS & 10 Fold CV & Naïve Bayes \\
\hline GW09 & Classifier Subset Evaluation & BFS & 10 Fold CV & Bagging \\
\hline GW10 & Classifier Subset Evaluation & BFS & 10 Fold CV & Boosting \\
\hline GW21 & Classifier Subset Evaluation & GSW & Full Training Set & Grid Search \\
\hline GW22 & Classifier Subset Evaluation & GSW & 10 Fold CV & Naïve Bayes \\
\hline
\end{tabular}




\subsection{Performance Evaluation Metrics}

The formula used for measuring classification accuracy is given in equation 1:

$$
\text { Accuracy }=\frac{T P+T N}{T P+T N+F P+F N}
$$

The accuracy and $\mathrm{KC}$ can be measured using the $2 * 2$ confusion matrix $[2,30]$ given in Figure 6.

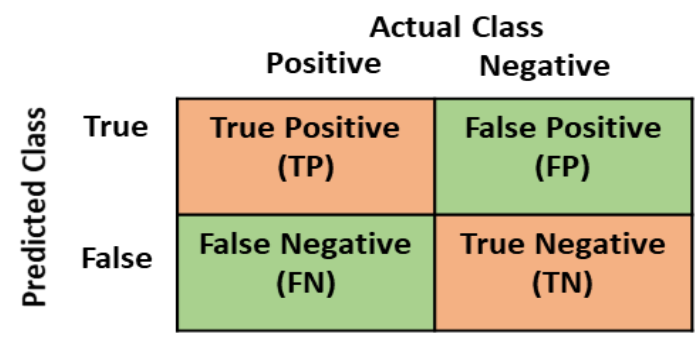

Figure 6. Confusion Matrix for evaluation of Performance Metrics

Using these two approaches we have generated around 84 models using two subset evaluators.

\subsubsection{Simple Wrapper Subset Evaluation (SWBE)}

In Weka, the WrapperSubsetEval technique is used with a Greedy Stepwise and Best First Search Method. It uses a learning approach, to evaluate the attribute sets and cross-validation is a technique used for estimating the accuracy of attributes [28]. Table 4 and 5 below mentioned shows the result generated these methods.

Table 3: Wrapper based Evaluation using BFS and 10 Fold CV

\begin{tabular}{|c|c|c|c|c|c|c|}
\hline \multicolumn{7}{|c|}{$\begin{array}{l}\text { Mean Shift Segmentation } \\
\text { Wrapper Subset Evaluation } \\
\text { Best First Search(10 FCV) }\end{array}$} \\
\hline Model No & Classification Algorithm & HF Subsets & CA & $\mathrm{KC}$ & Precision & Recall \\
\hline WWG15 & Naïve Bayes & HF3,HF4, HF6,HF7, H8,HF10 & $62 \%$ & $57 \%$ & $66 \%$ & $62 \%$ \\
\hline WWG16 & Bagging & HF1,HF2,HF5,HF6,HF7,HF10,HF12,HF13 & $64 \%$ & $59 \%$ & $65 \%$ & $64 \%$ \\
\hline WWG17 & Boosting & HF3,HF4,HF5,HF6,HF7,HF10,HF14 & $64 \%$ & $59 \%$ & $66 \%$ & $64 \%$ \\
\hline WWG18 & LIBSVM & HF4,HF6,HF8,HF9 & $60 \%$ & $54 \%$ & $63 \%$ & $60 \%$ \\
\hline WWG19 & $\mathrm{J} 48$ & HF1,HF4,HF5,HF6,HF11 & $62 \%$ & $57 \%$ & $63 \%$ & $52 \%$ \\
\hline WWG20 & Random Forest & HF2,HF3,HF4,HF6,HF10,HF12,HF14 & $68 \%$ & $64 \%$ & $70 \%$ & $68 \%$ \\
\hline WWG21 & Grid Search & HF3,HF5,HF6,HF10,HF11,HF12,HF14 & $55 \%$ & $48 \%$ & $59 \%$ & $55 \%$ \\
\hline
\end{tabular}

Observation Table 3: Shows the wrapper based Evaluation using Best First Search and 10 Fold Cross Validation as training module. Random Forest gives the best results i.e. CA-68\%, KC-64\%, Precision-70 $\%$, and Recall-68 \% shown in blue color with HF2, HF3, HF4, HF6, HF10, HF12, and HF14 as best subset of Haralick Features out of all. Boosting gives the second best result i.e. CA-68\%, KC-64\%, Precision-70 \%, and Recall-68 \% shown in blue color with HF2, HF3, HF4, HF6, HF10, HF12, and HF14 as best subset of Haralick Features out of all. The worst result was given by the Grid Search i.e. CA-55\%, KC-48\%, Precision-59\%, and Recall-55\%. 
Table 4: Wrapper based Evaluation using Greedy Step Wise and 10 Fold CV

\begin{tabular}{|c|c|c|c|c|c|c|}
\hline \multicolumn{7}{|c|}{$\begin{array}{c}\text { Mean Shift Segmentation } \\
\text { Wrapper Subset Evaluation } \\
\text { Greedy Step Wise (10 FCV) }\end{array}$} \\
\hline Model No & Classification Algorithm & HF Subsets & CA & KC & Precision & Recall \\
\hline WWG22 & Naïve Bayes & $\mathrm{HF} 3, \mathrm{HF} 4, \mathrm{HF} 6, \mathrm{HF} 7, \mathrm{H} 8, \mathrm{HF} 10$ & $62 \%$ & $57 \%$ & $66 \%$ & $62 \%$ \\
\hline WWG 23 & Bagging & HF 4,HF6,HF 7,HF 10,HF 12 & $63 \%$ & $58 \%$ & $65 \%$ & $63 \%$ \\
\hline WWG24 & Boosting & HF 3,HF 4,HF 5,HF6,HF 7,HF 10,HF 14 & $64 \%$ & $59 \%$ & $66 \%$ & $64 \%$ \\
\hline WWG25 & LIBSVM & HF4,HF6,HF8,HF9 & $60 \%$ & $54 \%$ & $63 \%$ & $60 \%$ \\
\hline WWG26 & $\mathrm{J} 48$ & $\mathrm{HF} 1, \mathrm{HF} 4, \mathrm{HF} 5, \mathrm{HF} 6, \mathrm{HF} 11$ & $62 \%$ & $57 \%$ & $63 \%$ & $52 \%$ \\
\hline WWG27 & Random Forest & $\mathrm{HF} 4, \mathrm{HF} 5, \mathrm{HF} 10, \mathrm{HF} 12, \mathrm{HF} 14$ & $66 \%$ & $61 \%$ & $68 \%$ & $66 \%$ \\
\hline WWG28 & Grid Search & $\mathrm{HF} 3, \mathrm{HF} 5, \mathrm{HF} 6, \mathrm{HF} 10, \mathrm{HF} 11, \mathrm{HF} 12, \mathrm{HF} 14$ & $55 \%$ & $48 \%$ & $59 \%$ & $55 \%$ \\
\hline
\end{tabular}

Observation Table 4: Shows the Wrapper Based Evaluation using Greedy Step-wise and 10 Fold CV as training module. Random forest and Grid search gives the best results for all the four parameters i.e. CA$66 \%, \mathrm{KC}-61 \%$, Precision-68\% and Recall-66\% shown in blue color. The best HF subset generated by it is HF4, HF5, HF10, HF12, and HF14.The second best result was given by the Boosting i.e. CA-64\%, KC$59 \%$, Precision-66\%, and Recall-64\% shown in pink color. The HF subsets generated by it are HF3, HF4, HF5, HF6, HF7, HF10, and HF14.The worst result was given by the Naïve Bayes algorithm i.e. CA-62\%, KC-57\%, Precision-66\%, and Recall-62\%.Figure 7 shows the identification of the suitable Haralick features for the particular type of defects using best first search and validation technique 10 fold cross validation.

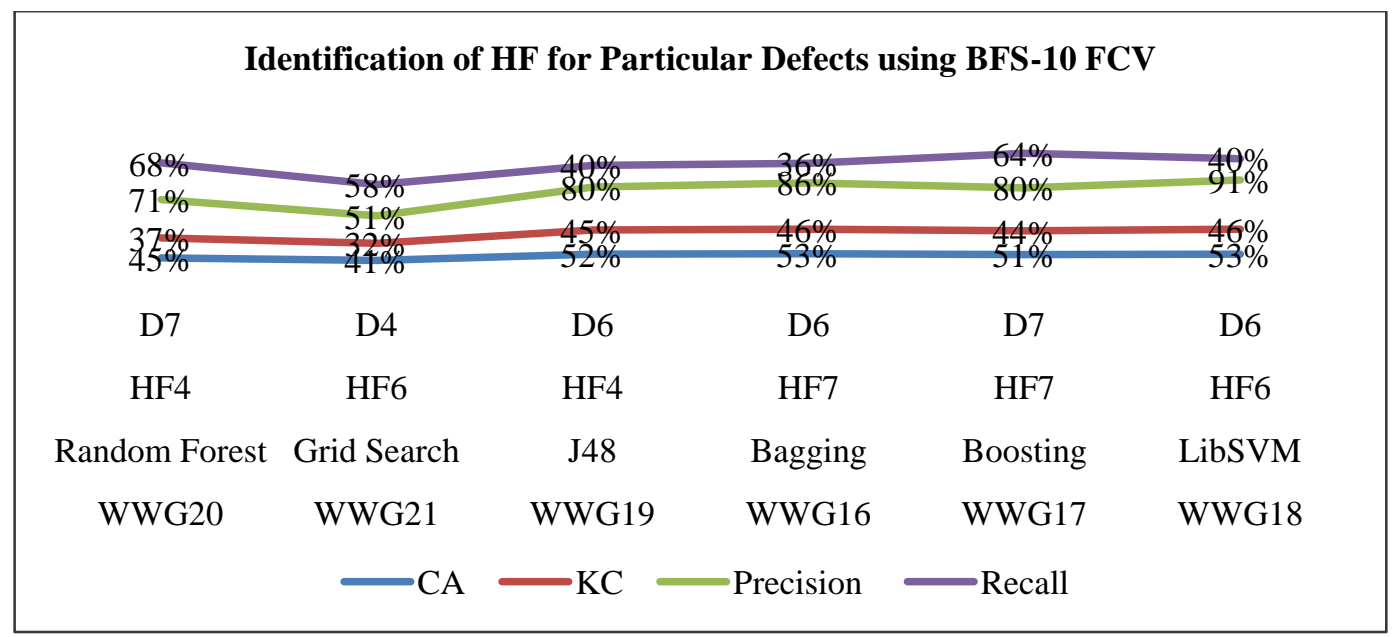

Figure 7 Identification of HF for particular Type of Defects using BFS-10 FCV

Observation Figure 7: The inferences drawn from figure 7 are as:HF4 (Variance) was able to identify the defects D7 (Camera Distortion) with CA-45\%, KC-37\%, Precision-71\% and Recall-68\% using Random Forest and D6 (Lightning Conditions) with CA-52\%, KC-45\%, Precision-80\% and Recall-40\% with J48 as Classification Algorithm.HF6 (Sum Average) was able to identify the defects D4 (Foreign Body) with CA-41\%, KC-32\%, Precision-51\% and Recall-58\%. Also HF6 was able to identify the defect D6 (Lightning Condition) with LibSVM.HF7 (Sum Variance) has identified D6 and D7 the particular type of defect using Bagging and Boosting as Classification Algorithm.

\subsubsection{Classifier Subset Evaluation (CSE)}

This method determines the importance of qualities based on their predictive capacity and degree of redundancy. Subsets with low inter-correlation but high correlations with the target class are favored. This attribute evaluator with the Best-First and Greedy stepwise is applied on the FVDB. The results obtained from this method are shown below. Table 7 shows the selection of best HF subsets using various classification algorithms for the silk fabric having eight types of defects present on it. 
Table 5: Classifier Subset Evaluation using Best First Search and 10 FCV

\begin{tabular}{|c|c|c|c|c|c|c|}
\hline \multicolumn{7}{|c|}{$\begin{array}{c}\text { Mean Shift Segmentation } \\
\text { Classifier Subset Evaluation } \\
\text { Best First Search(10 Fold CV) }\end{array}$} \\
\hline Model No & Classification Algorithm & HF Subsets & CR & KS & Precision & Recall \\
\hline WWG43 & Naïve Bayes & $\mathrm{HF} 3, \mathrm{HF} 4, \mathrm{HF} 7, \mathrm{HF} 8$, & $59 \%$ & $53 \%$ & $61 \%$ & $93 \%$ \\
\hline WWG44 & Bagging & $\mathrm{HF} 2, \mathrm{HF} 5, \mathrm{HF} 7, \mathrm{HF} 8, \mathrm{HF} 10$ & $63 \%$ & $57 \%$ & $64 \%$ & $63 \%$ \\
\hline WWG45 & Boosting & HF2,HF7,HF8,HF9,HF10,HF11,HF12,HF13,HF14 & $61 \%$ & $55 \%$ & $62 \%$ & $61 \%$ \\
\hline WWG46 & LIBSVM & $\mathrm{HF} 4, \mathrm{HF} 7$ & $59 \%$ & $53 \%$ & $61 \%$ & $59 \%$ \\
\hline WWG47 & $\mathrm{J} 48$ & $\mathrm{HF} 1, \mathrm{HF} 2, \mathrm{HF} 3, \mathrm{HF} 7, \mathrm{HF} 10, \mathrm{HF} 11, \mathrm{HF} 12, \mathrm{HF} 14$ & $64 \%$ & $59 \%$ & $65 \%$ & $64 \%$ \\
\hline WWG48 & Random Forest & HF1,HF7 & $55 \%$ & $49 \%$ & $55 \%$ & $55 \%$ \\
\hline WWG49 & Grid Search & HF1,HF7 & $59 \%$ & $53 \%$ & $62 \%$ & $58 \%$ \\
\hline
\end{tabular}

Observation Table 5: Shows the Classifier Subset Evaluation using Best First Search and 10 fold CV as training module. After Generation of best HF subset various classification algorithms were applied to obtain the results based on four performance metrics.J48 gives the best results i.e. CA-64\%, KC-59\%, Precision-65 \%, and Recall-64 \% shown in blue color with HF1, HF2, HF3, HF7, HF10, HF11, HF12, and HF14 selected as best subset of Haralick Features out of all.Bagging gives the second best result i.e. CA-63\%, KC-57\%, Precision-64 \%, and Recall-63\% shown in pink color with HF2, HF5, HF7, HF12, and HF14 as best subset of Haralick Features out of allThe worst result was given by the Naïve Bayes i.e. CA-62\%, KC-56\%, Precision-66\%, and Recall-61\%.Similarly, we have applied the same procedure using the greedy stepwise search method. The results generated by this method show that Random forest and Grid search gives the best results for all the four parameters i.e. 100\% using the full training set. The worst result was given by the Naïve Bayes algorithm i.e. CA-61.5\%, KC-56\%, Precision-66\%, and Recall-61\%. Using the 10 Fold CV method Boosting gives the best result i.e. CA-80\%, KC-77\%, Precision-82\%, and Recall-80\%. Bagging gives the worst result i.e. CA-62.5\%, KC-57\%, Precision-64\% and Recall-63\% with selected subsets HF2, HF5, HF7, HF8, HF10.

\subsubsection{Gabor Transform}

In Gabor Transforms, the feature extraction and selection process is based on a specific machine learning algorithm that we are trying to fit on a given dataset. The result obtained from various attribute evaluators are mentioned with their observations in table 8.

Table 6: Gabor Transform using Classifier Subset Evaluation using Best First Search and 10 FCV

\begin{tabular}{|c|c|c|c|c|c|c|c|}
\hline \multicolumn{8}{|c|}{$\begin{array}{c}\text { Gabor Wavelet Transform } \\
\text { Classifier Subset Evaluation } \\
\text { Best First Search (10 Fold Cross Validation) }\end{array}$} \\
\hline S.No & Model No. & Classification Algorithm & HF Subsets & CR & KS & Precision & Recall \\
\hline 1 & GW08 & Naïve Bayes & HF2,HF3,HF 5,HF6,HF 10,HF 12 & $31 \%$ & $22 \%$ & $30 \%$ & $31 \%$ \\
\hline 2 & GW09 & Bagging & $\begin{array}{l}\text { HF 1,HF 3,HF } 4, \text { HF 6,HF 7,HF 8,H } \\
\text { F9,HF 10,HF 11,HF 14 }\end{array}$ & $40 \%$ & $31 \%$ & $39 \%$ & $40 \%$ \\
\hline 3 & GW10 & Boosting & $\begin{array}{l}\text { HF 1,HF3,HF4,HF 7,HF 8,HF9,H } \\
\text { F10,HF11,HF12,HF } 13\end{array}$ & $39 \%$ & $30 \%$ & $39 \%$ & $39 \%$ \\
\hline 4 & GW11 & LibSvm & HF $2, \operatorname{HF} 7, \mathrm{HF} 10$ & $39 \%$ & $30 \%$ & $41 \%$ & $39 \%$ \\
\hline 5 & GW12 & $\mathrm{J} 48$ & $\begin{array}{l}\text { HF1,HF2,HF 4,HF6,HF 7,HF 8,H } \\
\text { F9,HF12,HF 13,HF } 14\end{array}$ & $40 \%$ & $32 \%$ & $41 \%$ & $43 \%$ \\
\hline 6 & GW13 & Random Forest & HF2 & $22 \%$ & $11 \%$ & $22 \%$ & $22 \%$ \\
\hline 7 & GW14 & Grid Search & $\begin{array}{l}\text { HF2,HF4,HF6,HF7,HF8,HF9,H } \\
\text { F10 }\end{array}$ & $35 \%$ & $26 \%$ & $35 \%$ & $35 \%$ \\
\hline
\end{tabular}

Observation Table 6: Shows the Classifier Subset Evaluation using Best First Search and 10 Fold Cross Validation as validation technique. J48 gives the best results i.e. CA-40\%, KC-32\%, Precision-41\%, and Recall-43 \% shown in blue colour with HF1,HF2, HF4, HF6, HF7, HF8, HF9, HF12, HF13 and HF14 as 
best subset of Haralick Features out of all. Bagging gives the second best result i.e. CA-40\%, KC-31\%, Precision-39 \%, and Recall-40 \% shown in pink colour with HF1, HF3, HF4, HF6, HF7, HF8, HF9, HF10,HF11, and HF14 as best subset of Haralick Features out of all.The worst result was given by Random Search i.e. CA-22\%, KC-11\%, Precision-22\%, and Recall-22\% with HF2.Similarly, we have applied the same procedure using the greedy stepwise search method. The results generated by this method show that J48 and Bagging gives the best results for all the four parameters i.e. CA-40\%, KC$32 \%$, Precision- $41 \%$ and Recall-43\% using the 10 Fold Cross validations. The worst result was given by the Random Forest Algorithm i.e. CA-22\%, KC-11\%, Precision-22\%, and Recall-22\%.

\subsubsection{Comparison of results between Generic Wrapper Based Approach and Gabor Transform}

The Figure 8 shows the comparison of Classification Accuracy with respect to Generic wrapper based approach and Gabor Transform. From the Figure 8 we can see that Generic Wrapper based Approach gives the best results as compared to Gabor Transforms. In this the $\mathrm{X}$ axis represents classification accuracy and $\mathrm{Y}$ axis represents the model number. Model number WWG47 gave the highest result of CA-64\% and selects the best subset HF1, HF2, HF3, HF7, HF10, HF11, HF12, and HF14 out of all. The best result given by the Gabor Transform was model number GW12 with CA- $40.4 \%$ and selects the best subset HF1, HF2, HF4, HF6, HF7, HF8, HF9, HF12, HF13, and HF14.

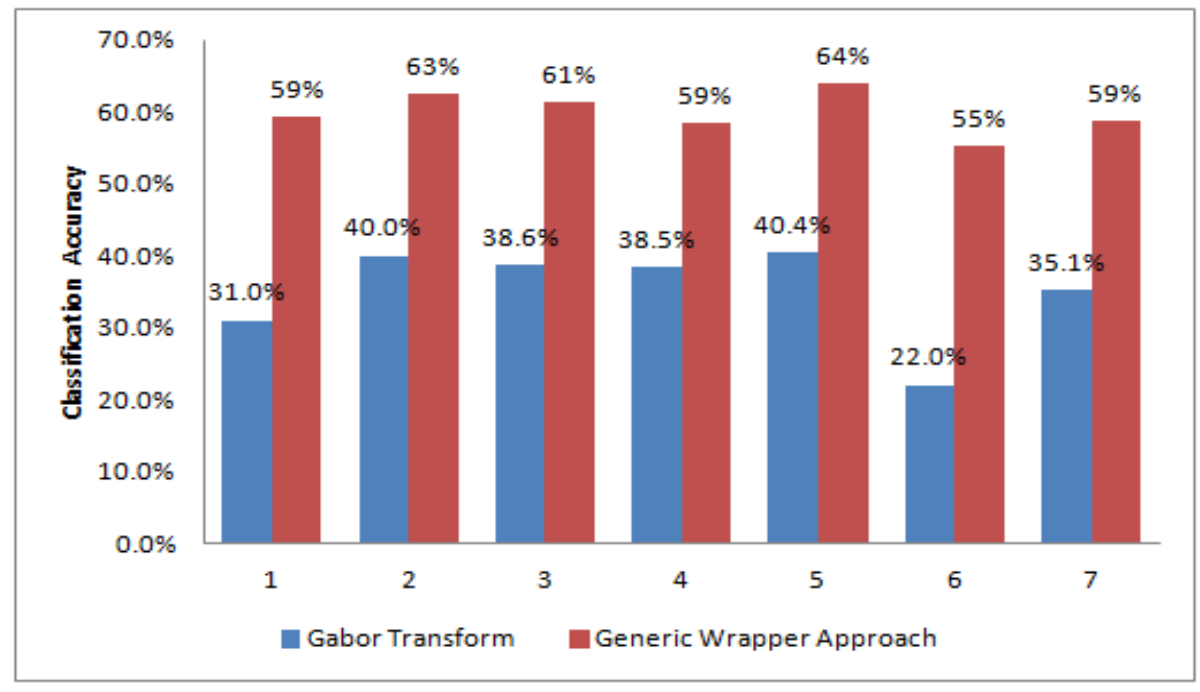

Figure 8 Comparisons between Generic Wrapper and Gabor Transform Approach

Model WWG45 using Boosting identifies the HF8 with defect D5 giving accuracy of $70 \%$. Also model number WWG44 was able to identify the suitable HF HF5 with defect D6 with accuracy 67\%. Similarly, in Gabor Transform model number GW12 was able to identify the suitable HF HF2 with defect type D7 with classification accuracy of 67\%. At the same time GW30 was able to identify the defect D6 with suitable Haralick Feature HF1 at 55\% of classification accuracy.

\section{CONCLUSION AND FUTURE SCOPE}

Various defect detection and classification models were designed. These models were executed on the dataset and the best models and best performers contributing Haralick features towards specific type of defect were noted down.

In simple wrapper-based approach model number WWG20 using random forest gives the best results for HF subset using 10 Fold CV. Values of the four parameters obtained in this are CA-68\%, KC$64 \%$, Precision-70\%, and Recall-68\%. It indicates that LibSVM was able to identify HF6 (Sum Average) 
suitable for Defect D6 (Lightning Condition) with CA-53\%, KC-46\%, Precision-91\%, and Recall-40\%. Similarly Boosting was able to identify HF7 (Sum Variance) for Defect D7 (Camera Distortion). The worst result was given by the Grid Search with an accuracy of $41 \%$ for HF6 for Defect D4 (Foreign Body). The experiment carried out helps to identify suitable Haralick features for some types of defects. Similarly, using Gabor Transform model number GW12 using J48 gives the best result out of all with CA-40\%, KC-32\%, Precision-41\% and Recall-43\%. Also, GW12 was able to identify HF2 (Contrast) as a best suitable defect to D7 (Camera Distortion) with CA 65\%. Similarly, GW30 was able to identify the defect D6 (Lightning Condition) for HF1 with CA of 55\%.

The limitation has been observed that this is not able to identify suitable Haralick features for all types of defects because it has higher chances of over-fitting and if the number of features increases it becomes computationally unfeasible and no guarantee of optimal solution is achieved.

In the future these defects and fabric types can be further experimented with and classified using various textural, colour, and shape features using various transforms.

\section{REFERENCES}

1. Ms. Shweta Loonkar, Dr. Dhirendra Mishra, Review of Back-propagation Algorithms for Defect Elimination with proposed DMASIC Methodology, (ICACCI) 5th International Conference on Advances in Computing, Communications and Informatics, IEEE Conference, $\mathbf{2 1}^{\text {st }} \mathbf{- 2 4}^{\text {th }}$ Sept 2016 LNMIT, Jaipur. http://ieeexplore.ieee.org/document/7732137/.

2. Ms. Shweta Loonkar, Dr. Dhirendra Mishra, Defect Classification for Silk Fabric Based on Four DFT Sector Features, IEEE Conference on Information and Communication Technology, 6-8 Dec 2019, Allahabad, India, INSPEC Accession Number: 19533717, https://ieeexplore.ieee.org/document/9066106.

3. Sodhganga Review of Literature-Defects.pdf

4. J. Escofet, R. Navarro, M. S. Millan, and J. Pladelloreans, "Detection of local defects in textiles webs using Gabor filters", Opt. Eng., vol. 37, pp. 2297-2307, Aug. 1998.

5. A. Kumar and G. Pang, "Fabric defect segmentation using multichannel blob defectors", Opt. Eng., vol. 39, no.12, pp. 3176-3190, 2000.

6. A. Kumar and G.K.H. Pang, "Defect detection in textured materials using Gabor filters", IEEE Trans. Ind. Appl., vol. 38, no.2, pp. 425-440, 2002.

7. A. Bodnarova, M. Bennamoun and S. Latham, "Optimal Gabor filters for textile flaw detection", Pattern Recognition, vol. 35, pp. 2973-2991, 2002.

8. Radovan Stojanovicw, Panagiotis Mitropulos, Christos Koulamas, Yorgos Karayiannis, Stavros Koubias, and George Papadopoulos, Real-Time Vision-Based System for Textile Fabric Inspection, Real-Time Imaging 7, 000-000 (2001) doi:10.1006/rtim.2001.0231, available online at http://www.idealibrary.com

9. Chandrashekar G, Sahin F, A Survey On Feature Selection Methods, Computer and Electrical Engineering, 2014, pg.16-28.

10. Saeys Y, Abeel T, Peer YV, W. Daelemans, Robust Feature Selection Using Ensemble Feature Selection Techniques, ECML PKDD 2008, p 313-325.

11. V. P. Shah, N. H. Younan, S. S. Durbha, and R. L. King, A Systematic Approach to WaveletDecomposition-Level Selection for Image Information Mining from Geospatial Data Archives, IEEE Trans. Geosci. Remote Sen, vol. 40, no. 4, pp. 875-878, Apr. 2007.

12. S. S Durbha and R. L King, Semantics-Enabled Framework for Knowledge Discovery from Earth Observation Data Archives, IEEE Trans. Geosci. Remote Sens., vol. 43, no. 11, pp. 2563-2572, Nov. 2005. 
13. Surya S. Durbha, Roger L. King, and Nicolas H. Younan, Wrapper-Based Feature Subset Selection for Rapid Image Information Mining, IEEE, Geoscience, and Remote Sensing Letters, VOL. 7, NO. 1, January 2010.

14. D.Mladenic, Feature Selection for Dimensionality Reduction, Lecture Notes on Computer Science, Subspace, Latent Structure, and Feature Selection, Statistical and Optimization, Perspectives Workshop, SLSFS 2005, 3940, Springer, Slovenia(2006)84-102.

15. R.O. Duda, P.E.Hart, Pattern Classification and Scene Analysis, John Wiley \& Sons, Chichester, 1973.

16. M.V.Susana, F.M.Mendonca, J.F.Goncalo, etal, Modified Binary PSO for Feature Selection using SVM Applied to Mortality Prediction of Septic Patients, Appl.SoftComput.13 (2003) 3494-3504.

17. Wu Y, Liu Y, Wang Y, Shi Y, Zhao X, JCDSA: A Joint Covariate Detection Tool for Survival Analysis on Tumor Expression Profiles. BMC bioinformatics. 2018; 19(1):187.

18. Yang R, Zhang C, Zhang L, Gao R, A Two-Step Feature Selection Method to Predict Cancer lectins by Multi-view Features and Synthetic Minority Oversampling Technique. Biomed Res Int. 2018.

19. Yifei Mao, Yuansheng Yang, A Wrapper Feature Subset Selection Method Based on Randomized Search and Multilayer Structure, Research Article, Hindawi BioMed Research International Volume 2019, Article ID 9864213, 9 pages https://doi.org/10.1155/2019/9864213.

20. http://lmb.informatik.uni-freiburg.de/resources/datasets/tilda.en.html 